\title{
5. Playful Haptics in Families
}

\begin{abstract}
In Chapter 5 we begin to focus upon the ways in which families "play" with co-presence through different engagements with digital media. We discuss in detail our methods around understanding co-present practices, especially through "tactile digital ethnography." This chapter examines how studying the hand gestures in, and around, the screen might help us contextualize a more embodied practice of mobile media in the everyday.
\end{abstract}

Keywords: play; families; ethnography; tactile; methods

For 6o-year-old Patricia Williams in Melbourne, locative media like Google Maps felt counter-intuitive when understanding place. The recent retiree found digital maps disorientating. She preferred to trace the paper map with her hand-as if the haptics (touch) of the hand could enact the movement of her legs. She found the pulling, pitching and zooming in and out confusing, creating a disembodying sense of scale and spatiality. However, Patricia actively engaged with social mobile media, especially the news.

Damien Williams, Patricia's 30-year-old son and Andrew, his 40-yearold partner, lived with Patricia in the same household. Andrew was the official IT person of the household, who installed and de-installed apps on everyone's mobile media. Both Damien and Andrew loved locative media and used it in many day-to-day scenarios. In particular, Andrew even had a dog locative media app that allowed him to track where the dog was in the house when he was at work - affording a particular type of care at a distance and friendly surveillance. Damien was an avid social media user who often tagged locations and time-stamps daily. His screens were dirty and broken from constant use and exposure.

This is in sharp contrast to Susan Jones whose screens were untouched by hands. Susan, a 65-year-old retiree who also lived in the house, was starting to get acquainted with mobile media by doing iPad classes. She would not

Hjorth, L., K. Ohashi, J. Sinanan, H. Horst, S. Pink, F. Kato, B. Zhou, Digital Media Practices in Households: Kinship through Data. Amsterdam: Amsterdam University Press, 2020 DOI 10.5117/9789462989504_CHO5 


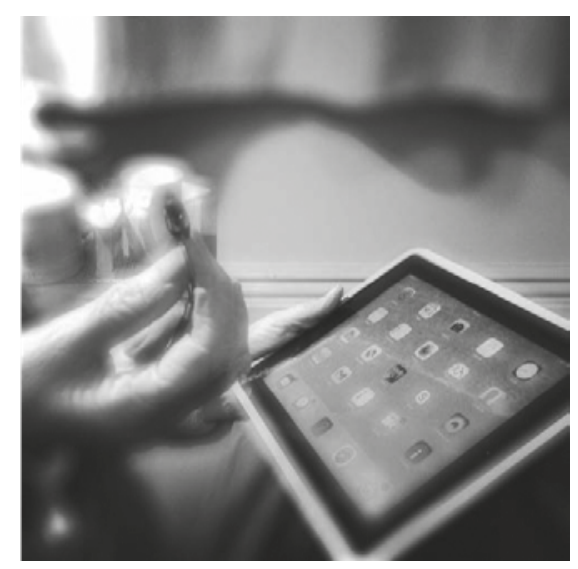

Figure 5.1: Susan using her iPad pointer (so her fingers don't touch the screen)

touch the screen with her hands, instead using a special pen pointer that she bought after seeing one of her iPad teachers using it (see Figure 5.1).

The "Williams" family is indicative of the multiple ways in which locative media, as part of broader social mobile media, was and was not being used in Melbourne households. We found generational and gendered differences playing out, both in and around their relationships to embodiment and notions of place. As Patricia's example highlights, the experience of place through paper maps creates a different sense of scale and movement that is constant, whereas the movement with Google Maps gives a sense of inertia and disorientation. Locative media use and non-use moves in and out of household rhythms - as symbolic of the screen's relationship to embodiment and place. For some participants, screens are so much part of our lives that they reflect the messiness of life through dirt and scratches. Other screens are viewed as a continuum of older screen practices whereby it was seen as bad to touch screens, such as TV screens.

The rise of haptic playful screens and media practices requires us to revise our methods. What does the dirty screen mean to a sense of embodiment? And does this differ, if at all, with the screen that remains untouched? What does it mean to engage with the intimate and haptic dimensions of media practice? What does it mean to study mobile media through knowing hands? What do the hands say that go beyond the spoken? Can we find similarities across cultural contexts? Or are there culturally specific gestures?

As we argue elsewhere, we need a theoretical interrogation of how we know through the hand, one which offers a deeper understanding of what and how we might learn through a tactile approach to digital ethnography (Keogh 2015). In other words, there is a growing need to understand screens 
as part of a haptic ecology that moves in, across and around the screen. We need to consider what researching through and by the hands might add to our methods and theorization for mobile media and screen research. These haptic practices inform how we interact, experience and understand locative media in our everyday.

In this chapter we develop this line of investigation through our crosscultural studies of locative media in and around the family. We consider how taking a tactile digital ethnography approach (Pink et al. 2016) might teach us new things about how we can study the practice through the "knowing" hands that might express tacit meanings. These meanings can be generational, technological affordances, culturally specific and also arguably innate gestures. In particular, we consider through this approach what "knowings" are culturally specific and which ones are more generic in and around the mobile screen. This study did not intentionally begin as a study of the hands, but through the digital ethnographic methods and the process of ethics where we wanted to de-identify our participants, filming in and around the hands and screen became increasingly prevalent.

\section{Reading Gestures}

Game studies have a long history in understanding the importance of touch as a sense of embodiment. As Brendan Keogh $(2015,1)$ observes in his phenomenology of videogame play, that there is a need to attend to the multi-sensorial dimensions of play through, in and around screenplay. Keogh argues, "Through an entanglement of eyes-at-screens, ears-at-speakers, and muscles-against interfaces players perceive videogames as worlds consisting of objects and actors with texture, significance, and weight" (Ibid.). Game consoles such as Nintendo Wii have been important in understanding and addressing the multi-sensorial experience of play in and around the screen-much of the work discussing this console speaks about the excessive and in-excessive deployment of the gesture (Giddings 2014). Simon (2009) argues that there is a "gestural excess" present in all videogame play that has been harnessed by haptic technologies such as Nintendo Wii and, more recently, touch screen play.

As the work of David Parisi (2009) discusses, it is important to create a media archeology of the touch in order to understand more deeply the relationship between touching and media. Touching did not just become important with smartphones. Parisi notes through the example of the Nintendo DS, the advertisements had to teach people to learn to touch the screen after decades of etiquette not touching the screen. It is important 
to remember this history when considering the normalizations around touching screens. They are generational and they are cultural. They are taught, untaught, retrained.

Parallels to the rise of the touch and screen-and its impact upon the spatial dynamics and context outside the screen/media-can be made with the rise of personal and portable technologies. For example, Rey Chow (1999) in her important study of the Sony Walkman in China argued that the technology revolutionized the role of listening. In China, listening had been historically a collective and public activity. Through the Walkman, it became internalized and personalized. Alternatively, Japan has a long history of the personal and portable as argued by Ito, Matsuda and Okabe (2005). The focus upon contemporary media as participatory needs to engage with the fact that participation takes many forms-including listening and the tacit.

The role of gestures in and around portable media has its history in newspaper and novel cultures. Reading traditionally in Chinese and Japanese required readers to begin at the Western "back" of the book and to move backwards to the front. In its adaption to touch screens (mobile and tablet) there has been some re-choreographing of the hand and reformatting of the book. In a western context swiping might involve the flicking from right to left for "page turning," which has then been adopted into eastern contexts. It is significant to recognize that these gestures are not innate but historically specific.

In this chapter we consider some of the ways in which haptic play moves in and around the attendant screen cultures. What we see is that the haptic quality of the screen creates different techniques and affective practices across cultures in the three locations Melbourne, Tokyo and Shanghai. We firstly define haptic play and its relation to the screen as defined through fieldwork. We see how affective labor through emojis can create different playful approaches to intimacy in and around the screen.

\section{Haptic Play and Screens}

It's a personal record. I enjoy the moment of check in. It's like treasure hunting. I would feel frustrated if I couldn't check in because of lack of WiFi connection. I don't want to socialize on Swarm. It's my own world. (22-year-old Yuto)

As Yuto noted, locative media game Swarm provided him with a playful way in which to think about his movements. He gained a sense of joy, much 


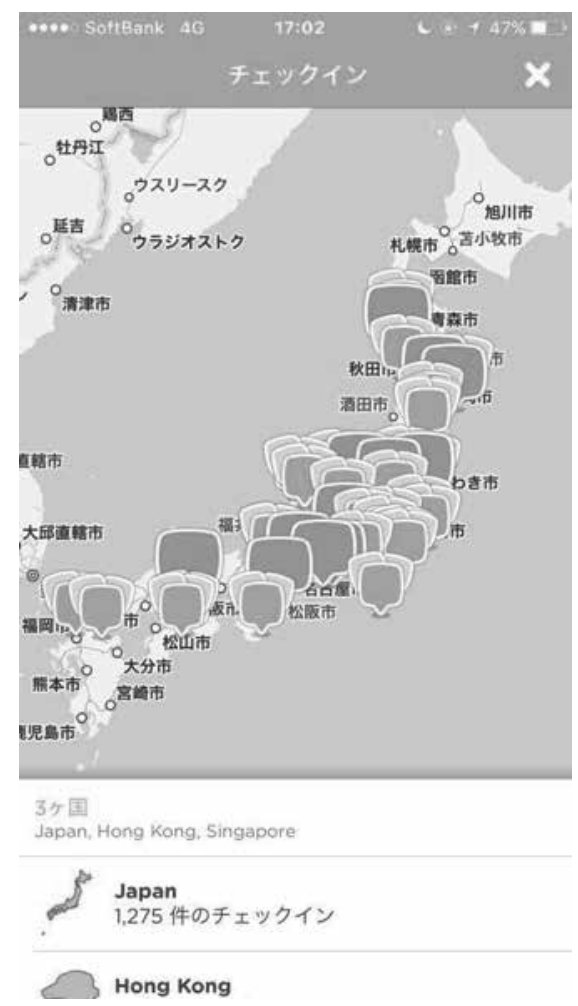

Figure 5.2: Yuto's journey via Swarm mapping

like a game reward, when viewing his journeys via Swarm. As a 22-year-old undergraduate student, Yuto moved from the northern part of Japan to Tokyo to enter the university. Yuto lived with his father (52-year-old), who worked in Tokyo, while the rest of his family - his mother (52-year-old) and his younger brother (17-year-old)—continued to live in his hometown. He bought his iPhone before entering the university and downloaded 10 apps including Twitter, Facebook, Instagram, LINE and Swarm.

Yuto used to tweet on a daily basis, but at the time of our research he did not tweet anymore because he had started job hunting and was worried some companies would judge him via his tweets. After that he made his Twitter account private. Yuto's media practices changed dramatically when he met his girlfriend and started to feel that he did not need to engage with social media, from then on only using it as a personal archive. Yuto's favorite app was Swarm, which he checked in on almost every day.

In the example of Yuto we see how media can be deployed in different ways to create a sense of haptic play, which moves in and around the screen (Figure 5.2). For Yuto, haptic play occurred through his deployment of Swarm 
as a form of a personal diary for mapping place. But it was also translated into other practices. For example, for Yuto, the sumaho (smartphone) was an extension of intimacy with his girlfriend, in the form of "girlfriend in the pocket." Yuto had his sumaho turned on and received alerts when his girlfriend sent him a message. He did not carry his sumaho in his hands but rather carried it in his pocket. These pockets become repositories for haptic play, awaiting his girlfriend's message. Haptic play moves in and around the screen and knowing hands.

Hjorth, Richardson and Balmford (2015) note in their study of mobile games how questioning seemingly trivial gestures in and around mobile media practices actually embodies a wealth of "deep" attitudes about privacy, boundaries between work and play, social etiquette, and care for the device. A "simple" gesture of having the phone screen face down when talking in face-to-face scenarios can reveal complex methods for negotiating engagement between online and offline worlds. As they note "it is clear that the bodily methods of interfacing with the materiality of the screen are of paramount importance to participants' engagement." The notion of haptic play seeks to engage with these new "techniques of the body" that must be accounted for if we are to interpret the complexities and intentionalities of use.

In the context of mobile touchscreens, it is not that such devices demand a more embodied or sensory mode of interaction, but that they have "alerted us to the sensoriality of our embodied and affective engagement with media in new ways" (Pink 2015, 6). As we explore the mutual imbrication of media interfaces and our embodied selves, the intimate connection between perception and meaning that is always-already both individual and collective.

Paterson $(2007,1)$ explores both the historical and contemporary theorizations and dimensions of touch. He argues that touch cannot simply be defined in physiological terms; it is also always "a sense of communication", and more significantly, it is manifold. This turn to "touch theory" is complexly interwoven with the emergence of haptic technologies and, in media studies, is often applied to the analyses of computer and touchscreen interfaces. In Paterson, Dodge and MacKian's (2012) terms, haptic media engage the manifold facets of touch, a sense ensemble that incorporates cutaneous, kinesthetic, proprioceptive, somatic, mimetic, metaphoric and affective modes of perception. Over the past decade such analyses of the haptic interface have enabled fertile connections between media theory and the disciplinary fields of sensory studies and new materialism. 
Parisi $(\mathbf{2 0 1 5}, 6)$, for example, traces an "intensified focus" in game studies on the way digital games invoke and depend on the body's movement and perception, which has followed the success of gesture controls and haptics (such as vibrating or rumbling controllers, or mimetic devices and applications such as the Nintendo Wii and many mobile games). In response, researchers have developed "nuanced theoretical frameworks capable of accounting for the body's newfound centrality to the play experience." Just as Parisi demonstrates the ways in which mobile gaming consoles advertisements sought to educate users about touchscreen interfaces after years of non-tactile screen cultures in the form of TV, so too can we see that the "intuitive" nature of touch and play is actually culturally and historically situated.

Sicart $(2014,22)$ distinguishes between play and playfulness. He has taken on the notion of "playfulness" as an attitude, an orientation to an activity, which also characterizes contemporary media. This attitude is not specific to games but can be seen in the seamless integration of games and creative mobile apps into our everyday lives and modes of communication, the perpetual expansion of participatory media forms, and in the rise of ever evolving social media services that enable users to upload, share and remix their own and others' creative "small media" content.

Exploring playful practices and the lusory attitude from the position of games gives us different ways to think through the role of data and algorithm in media cultures, from the quantified self $(\mathrm{QS})$ and gamification, to virtuoso play, creativity and playful resistance. Here the playful can be seen as an orientation to action, a mode of inquiry, and a set of practices that can help to expose some of the tacit power relations in and around the rhythms of data in everyday life. Play is fundamentally a creative, political and social activity.

\section{Haptic Rhythms}

Haptic interfaces can provide new experiences for and of the screen within everyday contexts. These haptic rhythms can create a sense of intimacy and temporal urgency that might not happen with non-haptic sense. Haptic screens provide a greater embodiment by deploying not only the sense of visual, but also, more importantly, the often-tacit sense of touch. The role of touching is culturally specific as well as historically informed. Touching involves a type of embodied sensorial experience. As noted with Yuto, haptic media in the form of vibrating alerts from the girlfriend in his pocket created 


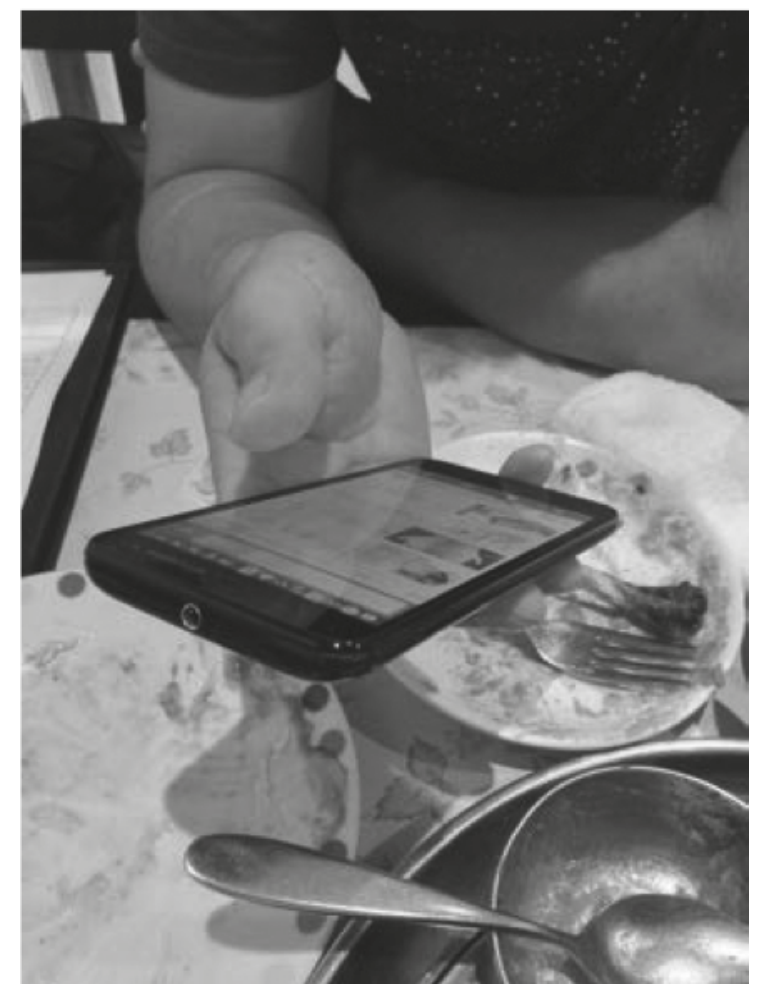

Figure 5.3: Yuto's smartphone was often in his pocket. He took it out during the interview.

a sense of intimacy as well as urgency (Figure 5.3). When Yuto received the vibration in his pocket he brought it out and quickly replied. Then he placed it back in his pocket. He was unaware of other people's messages as the phone was hidden in the pocket.

For Mana, the haptic nature of the mobile screen meant that she and her mum were so quick in their responses throughout the day that they were almost instantaneous. When Mana received the vibration that her mum had sent a message, she replied straight away. Mana had recently experienced some changes in her life that had made her exchanges with her mum even more frequent.

One year before our research, Mana broke up with her boyfriend of ten years. This made her quit her job in a local region and move to Tokyo for a new job. In order to try to "move on" from the heartbreak, Mana moved into a "social apartment" in the hope of meeting new people. A "social apartment," often referred to as "interactive rented accommodation" has a kitchen and lounge on the first floor where residents can contact and communicate 
with each other and associated individual rooms. Social apartments are a new phenomenon in Japan whereby companies buy an apartment and the employees can live there. They are, according to one advertisement, making "your life more open and connected." Here social refers to "social" in media rather than more historical or political notions of social. In places such as this, the friends become symbolic of the "family."

As Mana got used to the life in the apartment she began to think about the residents as akin to a family. As she described, "when I leave home they say, 'have a nice day' and when I go home, they say, 'welcome back'." Someone is always in the lounge. Someone receives a package when I am not there and if someone cooks or I cook, we eat together." Her "real family" lived in Tohoku (northeast) area. Her family included her parents, her elder sister, sister's husband, their child, her younger brother, brother's wife and their two children. As Mana lived apart from the rest of her family members, she made efforts to reply to messages on their LINE family group as soon as possible, using situational stamps such as "Cute!" or "Take care!" to create "a sense of participation." She bought different kinds of stamps to make her family laugh.

Takeshi, a 27-year-old hospital employee, lived with his family—his father (61-year- old), his mother (61-year-old) and his elder brother (31-year-old). He started to use a smartphone in 2012. His most used social media were LINE and Facebook, which he used to contact his friends. He also played games while using e-mail to communicate with his family. His favorite game at the time of our research was LINE PokoPoko puzzle game. In the game, the main character, Boni, and the other residents of Poko Forest must save the forest from the evil demons and return peace to their home by getting high scores on each stage and restoring the forest to its natural state by making the flowers bloom. The game, typical of the haptic play of contemporary mobile games, is as follows.

Each time you play the game, you consume one "clover." Clovers are like tickets necessary to play the game. Clovers regenerate at a pace of one every 30 minutes. Takeshi usually played the game for five minutes and consumed five clovers before going to work in the morning. By lunchtime, the clovers had regenerated. The rhythm of the game perfectly reflected his working hours. When we interviewed him in 2017, he had recently started to use another game app, Shogi Wars. Shogi is Japanese chess and in Shogi Wars, you can play with other players online. In Shogi Wars there is a "10SEC" mode that allows 10 seconds per move.

In Shogi Wars there is a "10SEC" mode that allows 10 seconds per move, which Takeshi played when he was with his family or his girlfriend after 


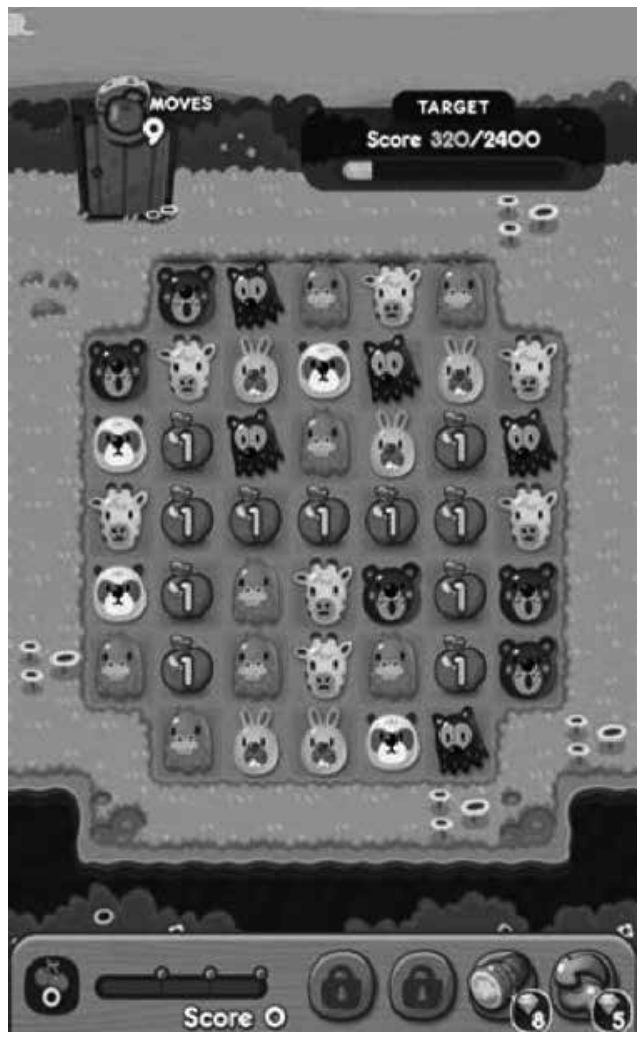

Figure 5.4: LINE PokoPoko

work. "In this mode, you can see the result of the match right away. So, it doesn't bother communication with others. I like to play this game under the gaze of my girlfriend," Takeshi explained. For Takeshi, it was his right thumb that was automatically deployed when using mobile touch screens. The impact of such haptic play exercise can be noted in the fact that his right thumb was bigger than his left thumb.

Within our Shanghai and Melbourne fieldwork, parallels and differences could be found. In Shanghai, unlike the traditional in-person family communication, family intergenerational communication via WeChat provided a more relaxing and playful format. The playfulness Sicart (2014) has suggested is prevalent in contemporary digital media is affording Chinese family's new ways of conversing in everyday scenarios. As parents of young adult children become more immersed within digital media and the attendant forms of unofficial literacies, the children are the ones being surprised by their parent's playful use of media. We see new forms of depth in terms of what haptic play performativity entails. 


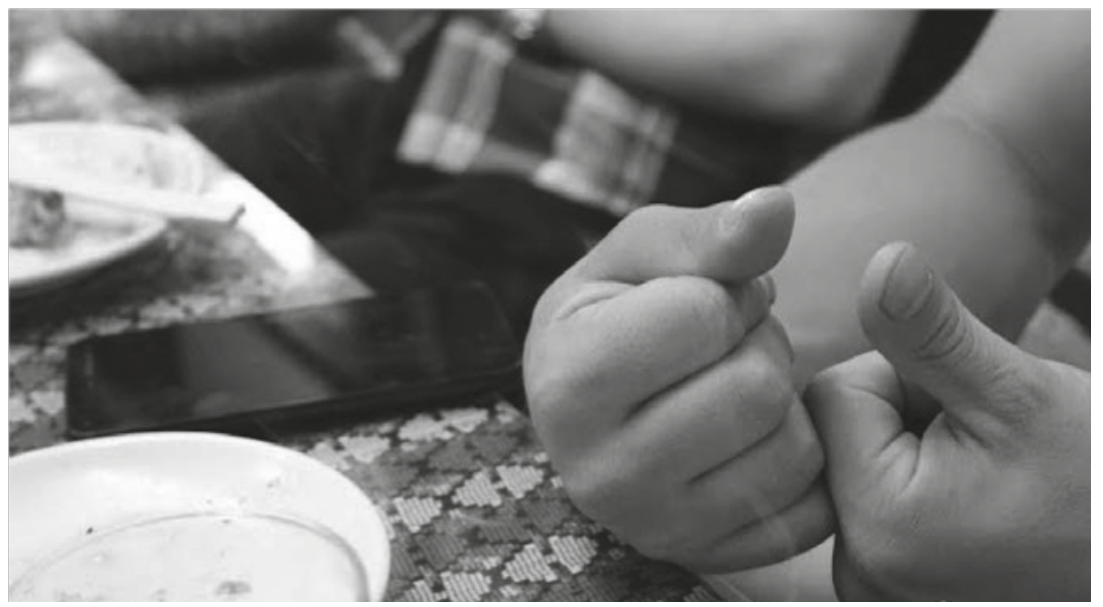

Figure 5.5: Takeshi's right thumb became bigger than his left thumb

\section{Haptic Play Poetry}

One such incident is the now famous case (see Figure 5.6) of a short poem written by a mother to her daughter on WeChat and then shared by the daughter on Weibo.com. During April of 2015, this WeChat screenshot rose to popularity and was shared by thousands on Chinese social media. The poem, taking the format of the WeChat message, roughly translates as,

Dear daughter Yaya/It's already spring time/Let's go on a trip/I'll go with you/and you'll bring the money/No matter where we go/Mount Tang-la is fine/the Desert Tengri is also good/Let's go on a trip at once/As long as we set off/I'll go withyou/andyou'll bring the money/YOU MUST REMEMBER TO BRING THE MONEY!!!

The daughter, Yaya, found the poem playfully humorous and so she posted it on Weibo (see Figure 5.6), which triggered a wide range of online responses. The Weibo post was forwarded over 19,00o times and the mother became famous, in an interesting way.

According to Yaya's other updates, her mother was very fashionable amongst her peers and was a savvy user of digital media like WeChat. The mother was very pleased about her impromptu internet fame and even has a post in her personal WeChat Moments to celebrate the phenomenon. This story is but one example of how WeChat's popularity across the generations is creating playful entanglements between generational differences in expectations and modes of use. In our interviews with parental participants, 


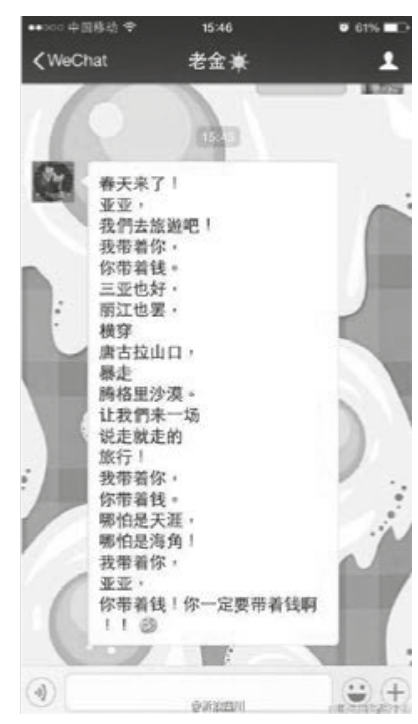

Figure 5.6: A mother's poem shared by her daughter on Weibo.com

"play" was the actual word (wán) used frequently to describe their daily involvement with WeChat. As a communication tool that connects friends and families, even strangers, together, WeChat was viewed as a useful "toy" to "play with." These digital immigrants explore WeChat in a playful way, enjoying sharing photos, sharing posts and sending stickers.

This playfulness is not only thanks to the rich media formats available on WeChat, but also due to plethora of interesting WeChat emoticons and stickers. There are two default kinds of emotional icons in WeChat - the old emotional icons derived from the first-generation of mobile social media, QQ, and emoji.

According to screenshots gathered from all participants, it became apparent that parents use the emoticons more often, while the children prefer using emoji. In addition, WeChat allows stickers that provide different ways to express feelings playfully. Here there are distinct parallels between generational use of WeChat in China and LINE in Japan. In WeChat there are two kinds of stickers - those provided by WeChat and users' customized stickers.

In Figure 5.8 we can see eight cartoon icons indicative of the WeChatprovided stickers. Within the WeChat sticker format there are those that are free and those that can be purchased. WeChat also encourages users to upload their customized stickers. In fact, customized stickers have gained much popularity among young WeChat users. Popular customized stickers change along with online hot topics. By using interesting stickers, dialogue on WeChat is textual, but also visual, emotional and playful. 


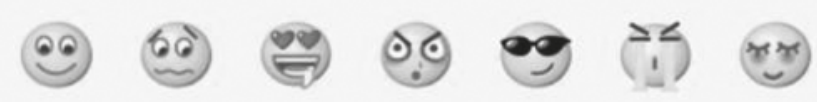

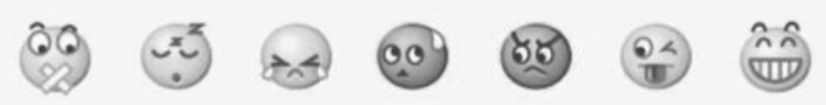

(30) :28) (20) $\times$

- 0 O 0

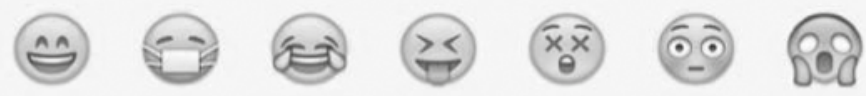

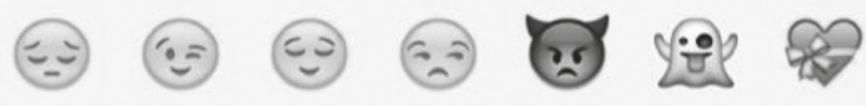

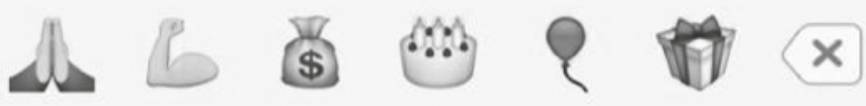

Figure 5.7: WeChat emoticons
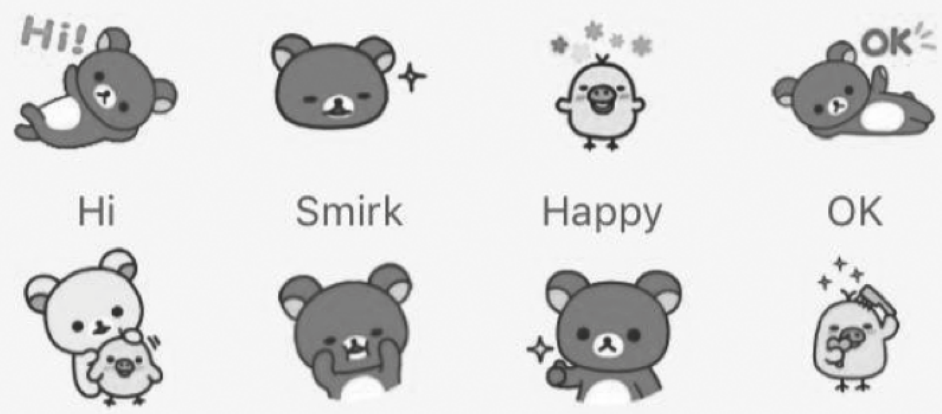

Smirk

Happy

OK

Praise
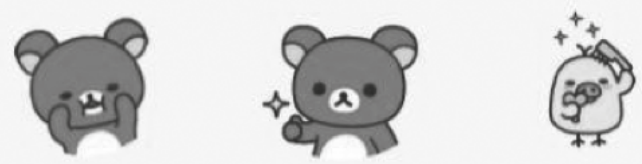

Silly

Good

Groom

Figure 5.8: An example of WeChat-provided stickers 


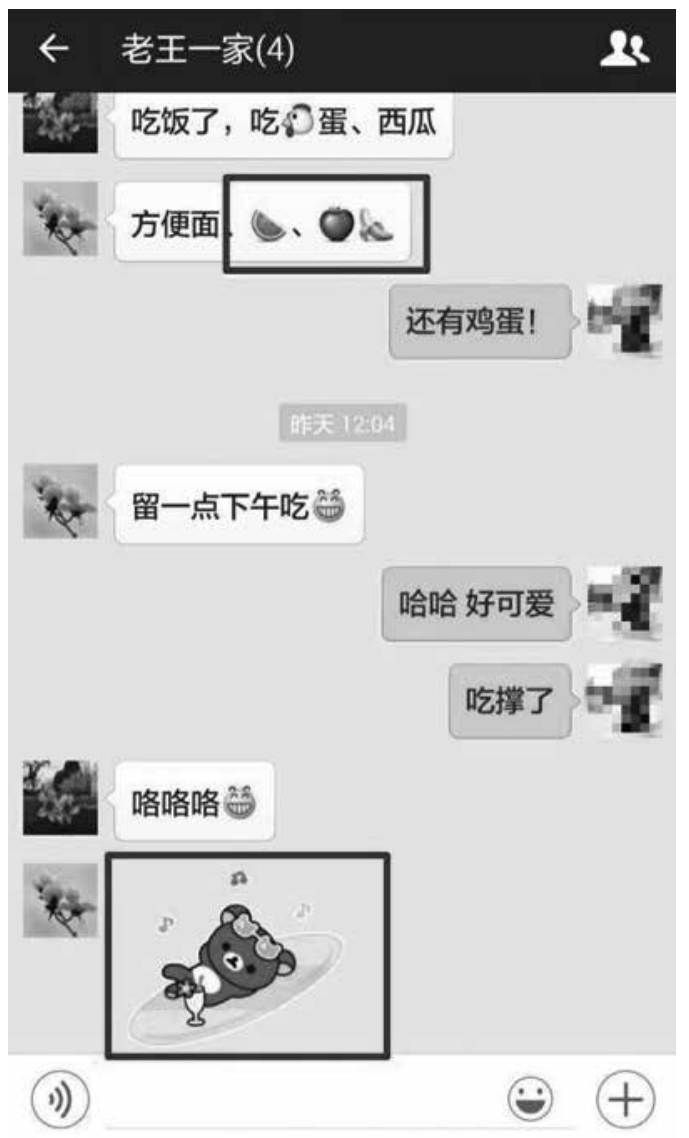

Figure 5.9: Screenshot of Taylor's family WeChat group

\section{Haptic Play Cadences (Co-present Frequency)}

In order to understand the emoticon phenomenon further, we examine the example of Taylor and her familial deployment of haptic play through frequent use of emoticons. A 24-year-old master's student living in Shanghai, Taylor left her remote hometown for University when she was 18. According to Taylor, WeChat had now become a significant communication channel for her family. She frequently used text, audio, and the Moments function in WeChat to interact with her parents. It was under Taylor's initiative that the family created a WeChat group for her parents to correspond with her frequently.

In Figure 5.9 we can see a screenshot from an interaction between Taylor's family members on WeChat. They were talking about lunch. Taylor's parents 


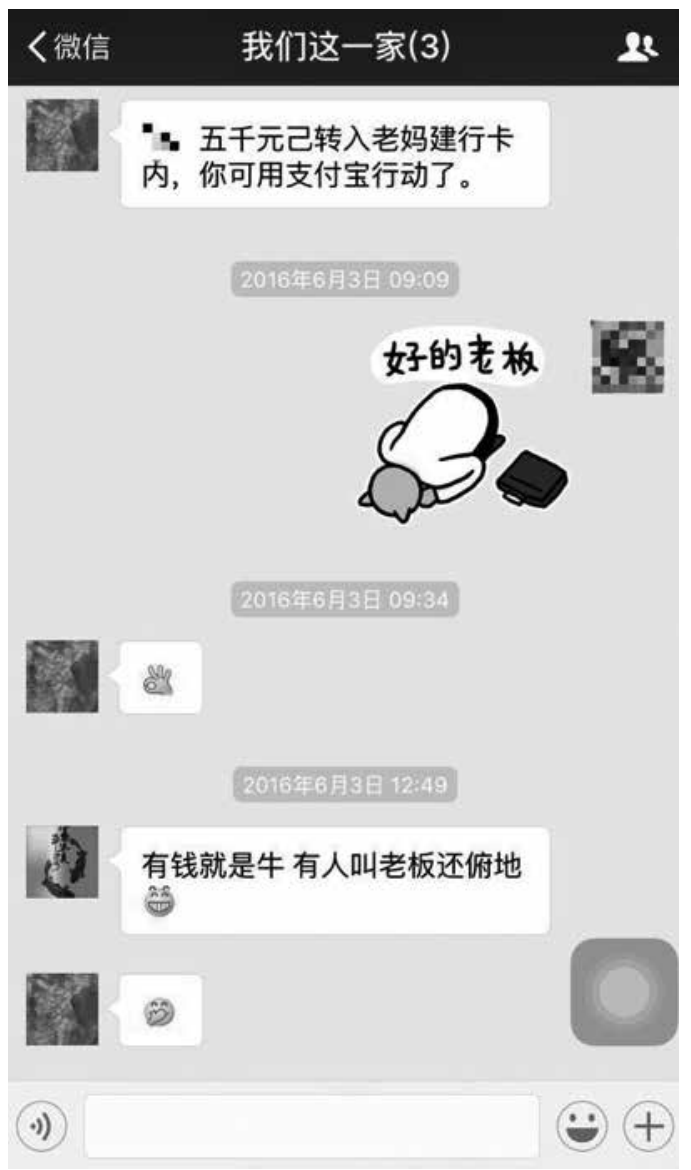

Figure 5.10: Screenshot of Tiffany's family WeChat group

deployed a range of emoticons and stickers to convey certain information and-more importantly - to express certain feelings. Through the use of a tapestry between emoticons, emoji and customized stickers, mundane familial interactions can be given a sense of emotional texture and haptic intensity.

In another case we find Tiffany. Tiffany is a 24-year-old master's student in Shanghai whose parents live elsewhere in China. She had also established a WeChat group for her family. According to Tiffany, though her parents did not have much knowledge of digital culture before she left, they had fully immersed themselves in the practice at the time of our research.

In Figure 5.10 we can see a screenshot of her parent's skillful and complex deployment of emoticons against Tiffany's minimal use of one sticker. What is interesting is that Tiffany chose a sticker meaning "OK, Boss!" however she 
was at first only able to express the meaning "OK." (When user types in "OK", this "OK, Boss!" sticker would automatically pop up as the recommended sticker, as long as the user has downloaded that set of stickers.) Tiffany noted, "It's really funny that my parents tried their best to understand the literal meaning of the sticker and replied with their illustration. That's really cute." As Tiffany's exchange with her parents demonstrates, phatic and mundane talk can be transformed into playful exchanges.

In Melbourne, we also found that family members of different generations can create a feeling of playfulness and closeness through using stickers and emojis. Nancy, who lived with her 6o-year-old husband and 30-year-old daughter in the outer western suburbs of Melbourne uses WhatsApp to chat with her cousin, nieces and nephews in Singapore a few days a week. She said, "I think they see a different side to me when we chat, it's not like I'm just the strict aunty when I visit." When her cousin or her niece wanted to have a more serious conversation with her, her cousin would Skype her and they would have a face-to-face conversation for up to an hour and her niece called on her iPhone using Skype or WhatsApp. For conversations that were more of a simple update or checking in, they would exchange quick texts over WhatsApp and Nancy always ended the exchange with a 'thumbs up,' high five or smiling emoji.

Nancy described how she picked up using emojis from her niece and nephew, since "they are always up to date with gadgets." For Nancy, WhatsApp was all about sharing emojis as part of an intimate co-presence. By using emojis she thought were cute, Nancy saw emojis as way of communicating intergenerationally - especially with younger members of her extended family in a way that was more familiar and colloquial to them (see Chapter 2). Through emojis she could create a relationship more akin to a friendship, rather than a strict aunty. Nancy's sister-in-law Esther lived 20 minutes from her with her husband, Patrick. Their daughter, Jasmine, lived with her husband in the inner northern suburbs of Melbourne. Jasmine saw her parents around once a week and the rest of the time, her primary communication with Esther was over Facebook Messenger.

Similar to Nancy, Esther was introduced to using emojis and stickers by a younger member of her family. Jasmine had been chatting to her mother and when she said she had to say goodnight as she had to wake up early in the morning, she followed the statement with a sticker from Facebook's Cutie Pet series of a cat crying. Esther exclaimed, "That's so cute! How do you do that?" and Jasmine explained how to access the sticker store. Within a few minutes, Esther had browsed the store and downloaded a few sheets 
of stickers. This type of action-based learning is synonymous with social media communication.

During our earlier visits to Esther, she commented that she rarely checked her phone when she arrived home from work-often missing calls and texts from Jasmine as her phone was still on silent from her workday. Esther's main reason for avoiding checking her devices at home was because she sat in front of a computer all day at work, so when she arrived home, the last thing she felt like doing was being tethered to yet another piece of technology. Jasmine also explained that she found contacting her mother extremely frustrating, as her mother never checked her phone. Given this issue Jasmine switched to Facebook Messenger as her primary source of contacting Esther.

Esther often ended her day by playing solitaire or reading on her tablet and when she turned it on, the tablet automatically updated her with notifications of messages received, so Jasmine was sure Esther would know when she had tried to contact her. Secondly, with stickers, Jasmine didn't just feel like she was overloading her mother with logistical information, about plans for the weekend or other kinds of coordination. By leaving a sticker at the end of the exchange, both mother and daughter felt like they had also left the conversation with something light-hearted, playful and cute.

\section{Conclusion: Playful Encounters}

As noted earlier, contemporary digital media has been defined as playful (Sicart 2014). Play and emotional gesturing can occur inside and outside the phone and is key to understanding the affective sharing of intimate co-presence. They help to reinforce and transform digital kinship ties. Through emojis, playful emotions can be shared without requiring faceto-face contact. Through emojis, intergenerational communication can be fostered in creative and inventive ways.

Both play and personalization have a particular genealogy in the mapping of digital media in the region. The role of personalizing digital media with emoticons (i.e. emoji) has a long history in Asian countries such as Japan, whereby the role of the cute (kawaii) plays into subcultural practices that seek to question traditional gender and age prescriptions, as noted in Chapter 2 and further discussed in this chapter. With the uneven uptake of mobile and locative media in the region, localized forms of cute character culture have become more prevalent across the generations. Grandparents, parents and children can be all found deploying emojis, emoticons and 
stickers in playful ways that are both tied to generational norms as well as providing new ways to transgress intergenerational boundaries.

In this chapter we have explored some of the ways in which haptic play is becoming a key part of everyday mundane intimacies between the generations. The affordances of the haptic and playful media like emoticons is creating ways in which generations are challenging norms around notions of media literacy. These are indicative of the types of digital kinship emerging in the three locations.

\section{References}

Chow, Rey. 1999. "Listening otherwise, music miniaturized: A different type of question about evolution." The Cultural Studies Reader, edited by Simon During. 382-399. London: Routledge.

Giddings, Seth 2014. Gameworlds. London: Bloomsbury.

Hjorth, Larissa, Ingrid Richardson, and William Balmford. 2016. "Careful surveillance and pet wearables: At home with animals.” The Conversation, 5 September. https://theconversation.com/careful-surveillance-and-pet-wearables-at-homewith- animals-63883. Accessed 20 May 2017.

Ito, Mizuko, Misa Matsuda, and Daisuke Okabe. eds. 2005. Personal, Portable, Pedestrian: Mobile phones in Japanese Life. Cambridge: The MIT Press.

Keogh, Brendan. 2015. "Paying attention to angry birds." The Routledge Companion of Mobile Media, edited by Gerard Goggin and Larissa Hjorth. 267-275. New York: Routledge.

Parisi, David. 2009. Game Interfaces as Bodily Techniques. New York: IGI Global.

- 2015. "A Counterrevolution in the Hands: The Console Controller as an Ergonomic Branding Mechanism.” Journal of Games Criticism 2 (1):1-23. http:// gamescriticism.org/articles/parisi-2-1. Accessed 20 May 2016.

Paterson, Mark. 2007. Senses of touch: Haptics, affects and technologies. London: Berg. Paterson, Mark, Martin Dodge, and Sara MacKian. 2012. "Introduction: Placing Touch within Social Theory and Empirical Study." In Touching Space, Placing Touch, edited by Mark Paterson and Martin Dodge, 1-28. Surrey: Ashgate Publishing.

Pink, Sarah. 2015. Doing Sensory Ethnography. London: Sage.

Sicart, Miguel. 2014. Play Matters. Cambridge, MA: The MIT Press.

Simon, Bart 2009. "Wii are out of control: Bodies, game screens and the production of gestural excess." Canadian Games Studies 3 (4): 1-17. http://journals.sfu.ca/ loading/index.php/loading/article/viewArticle/65. Accessed 26 May 2016. 